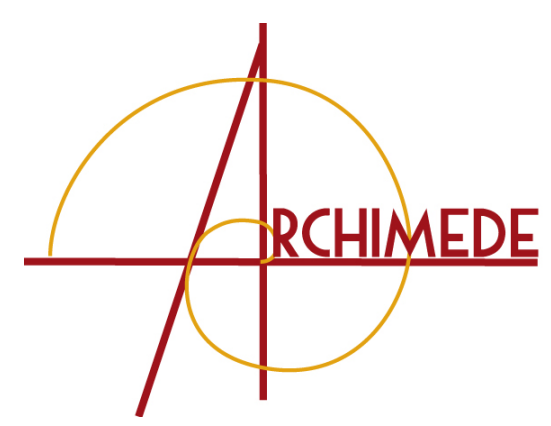

\title{
LA PRATIQUE DU RITUEL DANS LE TEMPLE ÉGYPTIEN
}

\section{Laetitia MARTZOLFF}

Docteur en égyptologie, chercheuse associée UMR 7044

Imartzolff@yahoo.fr

\section{RÉSUMÉ}

Le temple est le lieu par excellence du rituel. Les rituels qui y sont pratiqués sont de toutes sortes et conduits à fréquence variable. À côté du rituel divin journalier permettant de nourrir, soigner, parer et habiller le dieu quotidiennement, on observe tout un ensemble de rituels rattachés à des événements spécifiques de l'année comme le Nouvel An (rituel de I'Union au disque, rituel d'apaisement de Sekhmet) ou à des divinités/personnes particulières : rituel du mois de Khoïak pour régénérer Osiris, rituel de confirmation du pouvoir royal ou encore rituel de massacre des ennemis. Ces rituels sont principalement connus par des versions sur papyrus qui n'ont que rarement été découverts dans le contexte des temples et qui étaient d'abord des copies du texte rituel avant d'être des manuels de la conduite du rite. À ces sources écrites viennent s'ajouter une documentation iconographique pléthorique figurant sur les temples et le matériel issu des fouilles. Ce sont autant de documents qui doivent être pris en compte et confrontés afin d'obtenir une vision aussi complète que possible de la conduite des rituels dans les temples.
Temple is the place of the ritual. Rituals are of all kinds and performed to variable frequency. Next to the daily ritual made for feeding, adorning, or dressing divinities, we observe a whole set of rituals connected with specific events of the year, like the New Year (ritual of the disk Union for example), or conducted for special divinities / persons (ritual of the month of Khoiak's month, ritual of the confirmation of the royal power). These rituals are mainly known by textual sources on papyrus which were rarely discovered in context. They were textual versions of the ritual, more than manual for the priest. These written sources are completed with a plethoric iconographic documentation coming from temples and the material from the excavations. All these documentations (text, picture, material) must be taken into account and must be confronted with each other to obtain an idea, as complete as possible, of the ritual in temples and the way to perform it.
KeYWORDS 


\section{INTRODUCTION}

En Égypte, l'étude des rituels constitue un vaste champ de recherche qui peut s'appuyer sur l'existence d'un nombre très important de sources : en premier lieu, sur lestextes écrits sur papyrus ou tout autre support, ainsi que surles documentations archéologique et iconographique qui sont, en volume, tout aussi importantes. Le rituel est partout présenten Égypte: dans le temple certes, mais aussi dans la tombe et à ses abords, dans la sphère domestique, dans la Maison Royale. Nous avons choisi, dans le cadre du séminaire interdisciplinaire de janvier 2013 Archéologie du rituel, de nous pencher sur la question du rituel dans le contexte du temple. Il ne saurait être question d'être exhaustif - il serait utopique d'envisager de présenter en quelques pages l'intégralité des sources - ni même d'aborder les aspects théoriques de la conduite du rituel. Cette contribution n'a d'autre ambition que de proposer un aperçu des différentes sources permettant, dans le contexte spécifique du temple égyptien, de reconstituer un rituel en mettant l'accent sur les particularités de ces sources, leurs limites et certaines difficultés que pose leur interprétation.

Le temple est, par excellence, le lieu du rituel [1]. Les rites qui y sont pratiqués sont de diverses natures et menés à fréquence variable. Ainsi, le rituel divin journalier est conduit quotidiennement dans chaque temple, en théorie par le roi - premier prêtre d'Égypte -, en réalité par le prêtre principal. Les inscriptions du temple tardif de Dendéra, dont la décoration est datée de l'époque romaine, précisent que ce rituel était répété plusieurs fois dans la journée [2]. D'autres cérémonies sont rattachées à des périodes de l'année ou à des événements précis. Le rituel de l'union au disque est pratiqué en fin d'année, de même que le rituel d'apaisement de Sekhmet qui consiste à réciter la litanie des noms de la déesse afin de s'attirer ses faveurs pendant la période trouble du changement d'année [3]. Le mois de Khoïak, dernier mois de la saison de l'inondation, voit la conduite d'un ensemble de cérémonies en I'honneur du dieu Osiris alors que le

[1] TRAuneCKer 2005.

[2] Cauville 2000, p. X et 11-13. Les portes des chapelles C, D et E sont ouvertes trois fois par jour pour servir au rituel divin journalier

[3] Sur ce dernier point, voir Goyon 2006. mois suivant, le mois de Tybi est, dans certains temples, le moment des rites de couronnement du dieu et de la confirmation du pouvoir royal.

Les sources relatives à la pratique du rituel dans le temple sont, en plus des indices architecturaux [4], de diverses natures et peuvent être classées en trois grandes catégories : les objets servant à conduire le rituel ou témoignant de sa pratique, les sources textuelles indépendantes de la décoration du temple et les éléments de décoration puisque le temple égyptien offre cette particularité de présenter sur ses murs des images figurant des rituels. Au final, le volume de sources dont on dispose pour appréhender la pratique du rituel semble donc important. Si on ne peut nier le réel apport de l'existence de cette diversité de sources, il ne faut pas non plus ignorer certains problèmes.

Les objets témoignant des rituels dans le temple n'ont qu'exceptionnellement été retrouvés en contexte d'utilisation. Contrairement au domaine funéraire et en particulier aux tombes qui présentent du matériel en contexte primaire, les temples ont souvent continué à être utilisés bien après l'abandon des rites païens. Les objets du rituel qui sont parvenus jusqu'à nous sont ceux qui ont été cachés ou rangés, c'est-à-dire sortis du circuit de fonctionnement du temple. Il en est de même de la documentation liturgique papyrologique, qui nous est principalement parvenue par le marché des antiquités. Quant à la décoration des temples, elle s'inscrit dans I'espace de conduite du rituel, mais nous verrons que cette documentation doit être maniée avec précaution.

\section{LES OBJETS ET LE RITUEL}

Pour conduire un rituel, le prêtre se saisit de tout un ensemble d'objets ou plus exactement d'outils. On peut énumérer pêle-mêle les encensoirs, la vaisselle, les linges, les onguents, les éléments de parure, l'herminette, etc. Si tous ces objets sont intimement liés au rituel, ils se répartissent en deux catégories distinctes qui sont

[4] Nous n'aborderons pas, dans cette contribution, la question architecturale car il s'agit d'un aspect tout à fait spécifique qui nécessiterait un développement important. Il est évident que l'architecture du temple est en adéquation avec la conduite des rituels. 
généralement toutes deux représentées dans une cérémonie : objets rituels et instruments du rituel.

Les instruments du rituel sont les outils utilisés pour conduire le rite et qui ont la particularité d'être communs à plusieurs rituels et donc indépendants de la nature de ces derniers : ce sont, par exemple, les encensoirs ou les vases à purification. En effet, ces derniers peuvent être employés dans tous les rituels, dès qu'un état de pureté est exigé. C'est le cas, par exemple, dans le rituel de fondation du temple, de la confirmation du pouvoir royal ou encore du rituel divin journalier.

À I'inverse, les objets rituels sont les objets confectionnés et utilisés pour un rituel précis : I'herminette utilisée pour ouvrir la bouche dans le rituel du même nom en est un bon exemple. Quant à la statue de culte, support du divin dans le temple et objet du rituel «par excellence », elle est à la fois instrument du rituel car susceptible d'être utilisée pour plusieurs cérémonies et objet rituel puisqu'elle est employée au cours de plusieurs rituels.

La très grande majorité des objets rituels ou du rituel utilisés dans le temple ne nous sont pas parvenus, soit parce que le matériau employé était précieux - nombre d'objets étaient totalement ou partiellement en métal, qu'il s'agisse d'or, d'argent, ou d'alliage - , soit parce que I'histoire de l'édifice n'a pas permis leur conservation. Cela peut être dû, par exemple, à la transformation des lieux de culte en sanctuaires chrétiens ou à une réaffectation des espaces.

\section{Les objets rituels}

Il s'agit des objets spécifiquement rattachés à un rituel et le plus emblématique d'entre eux est la statue de culte. Point d'hiérophanie sur terre, objet habité par le dieu, la statue est le point de contact entre les mondes divin et terrestre [5]. Sa présence dans le temple fait de ce lieu un espace sacré. La statue abrite une manifestation du dieu qui doit être entretenue. C'est là la raison d'être du rituel divin journalier. Le naos qui abrite la statue est ouvert une, voire plusieurs fois par jour, afin de nourrir, habiller et parer le dieu. Les témoignages relatifs à ce rituel sont de deux ordres : textuels puisque le texte du rituel divin journalier est conservé sur le

[5] HELCK 1980 ; LORTON 1999.

[6] ROEDER 1914.

[7] Louvre D 30 mentionné avec photographie dans WIJNGAARDEN 1927 , p. 18 , fig. 12 . Voir également, pour une photographie couleurs, la Base Atlas du musée du Louvre : http://cartelfr.louvre.fr/cartelfr/ visite?srv=obj_view_obj\&objet=cartel_18944_56032_AE011322_ 01.jpg_obj.html\&flag=true (consultation juin 2014).

[8] Contardi 2009.

[9] BM EA37496 publié dans CuRTIS \& TALLIS 2005, p. 173 [266]. Voir également http://www.britishmuseum.org/research/collection_ papyrus Berlin 3055 ; iconographiques ensuite puisque les représentations des actions à conduire sont gravées sur les parois des temples.

Les statues de culte étaient conservées au sein des sanctuaires, dans des naoi dont plusieurs exemplaires sont préservés dans des musées [6]. À l'image du naos ptolémaïque du temple de Philae conservé au musée du Louvre [7], ces réceptacles peuvent être en pierre, mais aussi en bois. Leur décoration se limite, dans la majorité des cas, à la suggestion d'éléments architecturaux ou à des représentations symboliques du roi soulevant le ciel. Le naos de Séthi Irer conservé au musée de Turin fait figure d'exception car ses parois sont recouvertes des scènes et textes du rituel divin journalier [8].

Les naoi étaient le plus souvent fermés par des portes en bois afin de protéger la statue de culte. Ces portes n'ont que rarement été conservées même si nous en connaissons quelques-unes comme celle conservée au British Museum provenant d'un naos daté du règne de Darius Ier [9].

Quant aux statues de culte elles-mêmes, elles ne nous sont qu'exceptionnellement parvenues. Les inscriptions des temples nous indiquent parfois les matériaux qui les composent [10]. Il s'agit généralement de bois recouvert de métal précieux, or, argent ou alliage de type électrum. Plus rarement, il est question d'objets intégralement composés de métal comme la statuette d'or et d'argent d'Amon-Râ conservée au British Museum [11]. La nature des matériaux employés explique la rareté des pièces: elles ont été fondues ou démontées pour récupérer le métal précieux une fois leur utilité remise en question.

La découverte d'une statue de culte en contexte, c'est-àdire dans un sanctuaire et dans un naos, est plus exceptionnelle. En effet, cela suppose que l'activité du site ait été brutalement interrompue et le site figé à un instant précis. C'est effectivement ce qui a été observé dans le temple d'Osiris-Iou sur le site d'Ayn Manawîr, dans I'oasis de Kharga. En 1996, les fouilles ont mis au jour, dans une chapelle latérale, un ensemble de bois comportant une statue dans un naoset près duquel gisaient quelque 170 figurines en bronze, principalement des représentations d'Osiris. Les travaux ont démontré que la voûte de la pièce s'était effondrée vers 370 av. J.-C., figeant sur

online/collection_object_details/collection_image_gallery.aspx?asse $\mathrm{tId}=24411$ \&objectId = 111444\&partId = 1 (consultation juin 2014).

[10] C'est le cas par exemple dans les textes des cryptes du temple d'Hathor à Dendéra. Pour les inscriptions de ces espaces, voir Chassinat 1952 ; Chassinat \& Daumas 1965 et Cauville 2004.

[11] BM EA60006 publié dans QUiRKE \& SPENCER 1992, p. 76, fig. 55. Voir également http://www.britishmuseum.org/research/collection online/collection_object_details/collection_image_gallery.aspx?asse $\mathrm{tId}=973486$ \&objectId=154939\&partId=1 (consultation juin 2014). Cette statue a été découverte dans le temple de Karnak. 
place les éléments du rituel [12]. Si une telle découverte dans le contexte des temples reste exceptionnelle, la chose est plus fréquente dans un contexte funéraire. Les photographies de la tombe de Toutânkhamon en 1922 par $\mathrm{H}$. Carter en sont une bonne illustration [13].

Les colonnes 26 à 32 du papyrus Metropolitan Museum of Art 35.9.21, plus connu sous le nom de papyrus d'Imouthès, détaillent les paroles à prononcer lors de la conduite du rituel de Lancer les quatre boules [14]. Il s'agit d'un rite destiné à protéger le dieu Osiris de ses ennemis au moment de sa résurrection en envoyant aux quatre points cardinaux quatre boules d'argile (bnn.t $s \mid n$ ) inscrites au nom de divinités protectrices [15]. La publication dece papyrus a permis de comprendre la fonction de petites boules d'argile inscrites qui avaient été découvertes dans des contextes osiriens et dont la fonction restait inconnue. Le musée du Louvre en possède plusieurs lots qui ont généralement été découverts dans des tombes [16]. Plus récemment tout un ensemble de boules et de boîtes les contenant a été mis au jour dans les catacombes osiriennes d'Oxyrhynchos [17].

Les boules inscrites sont des preuves archéologiques de la pratique de ce rituel connu principalement par une version écrite. La découverte de ce matériel dans des tombes ou encore des complexes osiriens spécifiques comme les catacombes démontre que ce rituel était conduit dans un contexte funéraire et en rapport direct avec la nature du dieu Osiris, dieu des morts. Le rite trouve également sa place dans les temples quoique les représentations soient extrêmement rares et réservées à des espaces teintés d'un fort contexte osirien. C'est ce qui apparaît sur le linteau de la chambre $\mathrm{E}$ de l'édifice de Taharqa à Karnak : le rite est signifié par la représentation du roi en train de jeter les quatre boules [18]. Le texte précise que le rite n'est pas fait au bénéfice du dieu des morts, mais pour le dieu Amon d'Opê qui passe sur la tombe d'Osiris. Sur les murs latéraux de la chambre principale de la chapelle osirienne du temple d'Hibis ( $\mathrm{H} 2)$, les textes mentionnent les quatre déesses Ouadjet, Neith, Sekhmet et Bastet qui interviennent dans le rituel des quatre boules, mais il n'y a aucune image du rituel [19].

[12] Wuttmann 1995, p. 393-402, et Wuttmann, Coulon \& Gombert 2007.

[13] L'intégralité des photographies prises par H. Burton suite à la découverte de la tombe de Toutânkhamon est disponible pour consultation sur le site du Griffith Institute (http://www.griffith. ox.ac.uk/discoveringTut/, consultation juin 2014). Voir par exemple le cliché P1169.

[14] Goyon 1999. Le rite est également connu par d'autres sources qui n'offrent toutefois pas une version aussi complète que celle du papyrus Metropolitan : il s'agit des papyrus Brooklyn 47.218.138 et BM10288.

[15] Goyon 1975, p. 359-399, et Goyon 1999, p. 63-73.
La cuve à germination ou cuve à Osiris végétant, parfois aussi appelée cuve-jardin, est un autre exemple intéressant d'objet rituel. Il s'agit d'une cuve en argile prenant la forme d'un Osiris momifié dans laquelle des grains d'orge sont placés pour germination à l'occasion des cérémonies du mois de Khoïak [20] (Fig. 1) [21]. Les textes tardifs des chapelles osiriennes orientales du temple de Dendéra nous renseignent sur le déroulement des fêtes qui se tenaient du 12 au 30 Khoïak et en particulier sur la confection de figurines d'Osiris en terre [22]. Le 12 du mois, la cuve à germination était remplie de terre et la terre ensemencée pour la confection de la

Figure 1

cuve à germination, Institut d'Égyptologie de Strasbourg (n IES MA 798). Cliché Cassandre Hartenstein @) IES

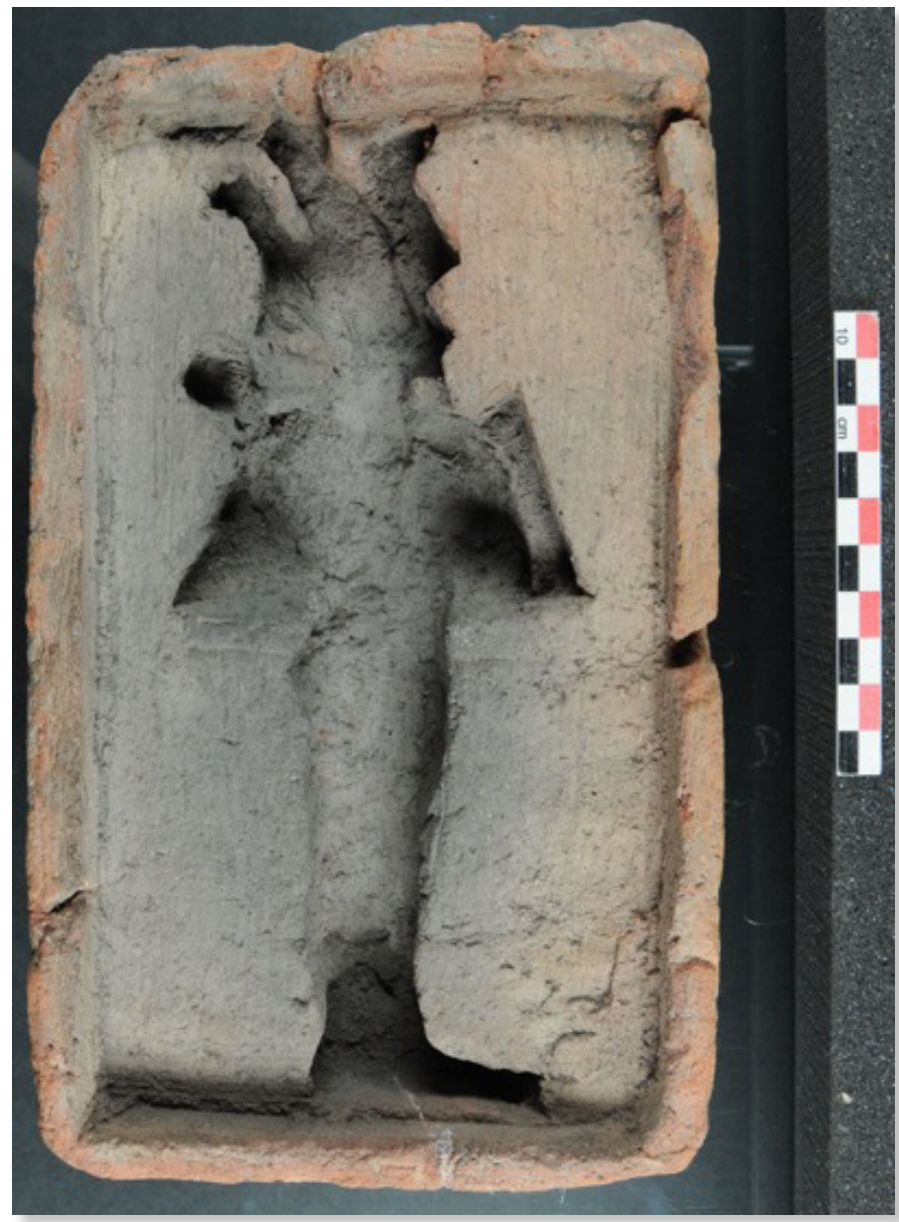

[16] Cf. infra, note 26.

[17] Sur ces constructions, cf. infra, notes 24-25.

[18] Parker, LeClant \& Goyon 1979, pl. 25.

[19] DAVIES 1953, p. 20 et pl. 20 (mur est, premier registre).

[20] Sur les cuves d'Osiris végétant, voir SEEBER 1980 et ANDREU 1997, et sur I'utilisation des cuves jardin, CHASSINAT 1968, p. 810-811.

[21] No IES MA 798. Je tiens à adresser mes sincères remerciements à Frédéric Colin, directeur de I'Institut d'Égyptologie de Strasbourg, qui m'a autorisée à citer et utiliser cet objet issu de la collection strasbourgeoise.

[22] ChASSINAT 1968, p. 819-823. 
figurine de Khentyimentyou, autre nom d'Osiris. La terre était arrosée jusqu'au 21, date à laquelle les figurines étaient « démoulées » avant d'être séchées au soleil et apprêtées pour les cérémonies ultérieures. Le 22, les figurines confectionnées étaient sorties en procession, alors que le 24, la figurine de I'Osiris de I'année précédente qui avait fait l'objet d'un culte était ensevelie.

Ces cérémonies du mois de Khoïak étaient célébrées dans les temples, en particulier dans les chapelles dédiées au dieu Osiris [23]. Le matériel archéologique témoignant de ces festivités n'a toutefois pas été découvert dans les temples. Il a plus souvent été mis au jour dans des structures appelées « catacombes osiriennes ». Il s'agit d'aménagements à l'architecture tout à fait singulière comprenant de grands couloirs dont les parois étaient de part et d'autre percées de petites niches. Deux aménagements de ce type ont été reconnus à ce jour : le premier a été découvert en 1993 à Karnak et consiste en un réaménagement sous Ptolémée IV d'une structure plus ancienne. On y observe trois couloirs parallèles comprenant au total 720 niches [24]. Le second, mis au jour en 2000,a été identifié sur le site d'Oxyrhynchos. Cet aménagement est contemporain des règnes de Ptolémée VI, VIII et Ptolémée IX Sôter II. Là, ce sont au moins deux salles d'une trentaine de niches qui ont été découvertes [25]. Dans chaque niche, la figurine d'Osiris de l'année précédente était ensevelie, en plus d'autres objets qui étaient utilisés pour différents rituels de protection. Les catacombes oxyrhynchites ont fourni plusieurs lots de boules d'argile utilisées pour le rituel du lancer des quatre boules [26]. La niche était fermée à la fin des festivités par une dalle sur laquelle était inscrite la date de l'ensevelissement [27].

L'herminette fait également partie de ce matériel rattaché spécifiquement à un rituel, celui de l'ouverture de la bouche, attesté d'abord dans le contexte funéraire et pratiqué sur la momie du défunt avant sa mise au caveau. Par cette action, le défunt pouvait reprendre possession de son corps après les opérations de momification [28]. Ce rituel est figuré dans les tombes à partir du Nouvel Empire ou retranscrit sur des papyrus accompagnant le défunt dans son voyage dans l'au-delà. Les outils servant à l'ouverture de la bouche ont été découverts dans les tombes et en particulier des exemplaires d'herminette. C'est avec cet objet de bois au bout recourbé que le prêtre touchait les orifices de la momie (nez, oreilles, bouche)pour que le ba du défunt puisse réintégrer son corps et que le mort retrouve ses capacités vitales (audition, respiration, alimentation...) [29]. Ce rituel était également conduit dans les temples sur les statues divines nouvellement confectionnées afin de permettre au ba du dieu d'en prendre possession. Dans les temples, la représentation de l'acte d'ouverture de la bouche au moyen de l'herminette, à l'image de ce qui est observé dans le contexte funéraire, est très rare et seuls deux exemples sont connus, l'un sculpté sur les parois du sanctuaire décoré par Thoutmosis III et usurpé par Alexandre à Karnak, l'autre dans la chambre $\mathrm{F}$ du grand temple d'Horus à Edfou [30].

\section{Les instruments du rituel}

L'entretien de la statue et la conduite des rituels nécessitaient l'emploi de tout un matériel, d'un ensemble d'outils parmi lesquels on peut mentionner les encensoirs en bois ou en métal qui servaient aux rites de fumigation pour purifier l'air et contenter les dieux en leur faisant respirer des odeurs agréables [31]. Les bas-reliefs montrent le roi en train de brûler de l'encens devant les divinités [32] et de nombreux exemplaires d'encensoirs en bois ou métal sont conservés dans les musées [33].

Les rites de purification nécessitaient également une vaisselle métallique figurée sur les reliefs et dont plusieurs exemplaires nous sont parvenus [34]. Enfin, les sistres
[23] Les plus célèbres sont celles du toit du temple de Dendéra (CAUVILlE 1997) mais on peut également mentionner celle du toit du temple d'Isis à Philae. Sur cette dernière, voir BÉNÉDITE 1893 p. 119-127, pl. 35-42, et pour des photographies récentes, HöLBL 2007, p. 71-77.

[24] Coulon, Leclère \& Marchand 1995 ; Leclère 2002.

[25] AMER 2012.

[26] Voir par exemple les exemplaires de boules conservés au musée du Louvre: http://cartelfr.louvre.fr/cartelfr/visite?srv=obj view_obj\&objet=cartel_19799_31572_e0029114.001.jpg_obj. html\&flag=true (consultation juin 2014)

[27] En fait, I'année de règne du pharaon était précisée dans une inscription courant sur le linteau de la niche, alors que le jour exact était précisé sur la plaque servant à fermer la niche. Voir Coulon 2011, p. 81-89.

[28] OтTо, 1960 ; QUACK 2005.

[29] Voir par exemple le lot d'objets reconstitué présenté au musée du Louvre :http://cartelfr.louvre.fr/cartelfr/visite?srv=obj view_obj\&objet=cartel_19167_27611_ev027085.001.jpg_obj. html\&flag=true (consultation juin 2014).

[30] Respectivement Porter \& Moss 1972, p. 119, n 397, et Nelson 1944 , p. 51 , fig. 6 ; ChASSinat 1928b, pl. 23a. On connait toutefois de nombreuses scènes d'offrande de l'herminette à une divinité sans que la mention de l'ouverture de la bouche ne soit faite dans le titre de la scène. Dans ces cas, c'est l'outil qui est présenté à une divinité, mais en aucun cas la pratique d'un rituel. Voir BEINLICH 2008, vol. 1, p. 329-330.

[31] Goyon 1984 et Meeks 1976.

[32] Par exemple, dans le temple d'Abydos: Calverley, Broome \& GARDINER 1933, pl. 7.

[33] Les exemples sont nombreux. Voir, par exemple, I'encensoir en métal E 13531 du musée du Louvre (http://cartelfr.louvre.fr/cartelfr/ visite?srv=obj_view_obj\&objet=cartel_3862_55864_AE006511_ 01.jpg_obj.html\&flag=true, consultation juin 2014).

[34] Calverley, Broome \& Gardiner 1933, pl. 7 ;Traunecker 1972, p. 205;BISSING 1901, n³426. 
peuvent également être rangés dans la même catégorie. Les cliquetis métalliques qu'ils émettaient devaient éloigner les ennemis et contenter les divinités [35].

\section{LES SOURCES EXCLUSIVEMENT TEXTUELLES : LES PAPYRUS}

La grande majorité des papyrus rituels conservés dans les musées a été acquise sur le marché des antiquités, notamment au XIX ${ }^{e}$ siècle. Le contexte précis de leur découverte est donc souvent resté inconnu, à l'exception notable de sites comme Soknepaiou Nesos et Tebtynis qui ont livré une multitude de papyrus en contexte, papyrus qui sont disséminés aujourd'hui dans tous les musées européens [36].

Les rituels pratiqués dans les temples étaient inscrits sur papyrus. Les compositions qui avaient souvent une origine très ancienne étaient copiées, complétées, parfois en partie réécrites pour être transmises dans les différents centres de culte par l'intermédiaire des Maisons de vie, sortes de conservatoire du savoir liturgique [37]. Dans les temples, les textes rituels étaient conservés dans les «maisons du livre (per-medjat - pr-md $3 . t$ )», autrement dit des bibliothèques [38]. Préservés dans des coffres, les papyrus étaient sortis ponctuellement pour être utilisés au gré des besoins dictés par la liturgie du temple. Les murs de la bibliothèque du temple d'Horus à Edfou portent les inscriptions des titres des ouvrages ou rituels qui y étaient stockés. Il y est fait mention du « Livre pour abattre Seth », du «Livre de protection du trône », du «Livre de protection des heures » ou encore du «Livre d'apaiser Sekhmet » [39]. Dans le temple d'et-Tôd, on trouve mention du «Rituel de remplissage de l'œil oudjat », du «Rituel de nouage de l'amulette » ou encore du «Livre pour rajeunir Horus comme Khepri » [40].

Les papyrus sont une source d'information incontournable pour les rituels car ils nous en communiquent le contenu, la raison d'être ou encore la fréquence de déroulement. Mais si les papyrus sont essentiels pour la conduite d'un rituel puisqu'ils contiennent une version des formules à réciter, ils ne sont que rarement un manuel pour le prêtre ritualiste définissantsa gestuelle ou ses postures dans la conduite de l'action rituelle.
Prenons comme exemple le rituel divin journalier du papyrus Berlin 3055 ou plus exactement, selon le titre de l'ouvrage, les« Formules des rites divins faits pour la demeure d'Amon-Râ, le roi des dieux, tous les jours, par le grand prêtre qui est en fonction. » [41]. Le papyrus regroupe 65 chapitres nécessaires à la conduite du rituel journalier en I'honneur d'Amon-Râ. On pourrait penser qu'il s'agit d'une présentation chronologique des actions à effectuer et que le déroulement du rituel suit l'ordre des chapitres. Pourtant, plusieurs indices vont à l'encontre de cette hypothèse comme la répétition de sections entières - je pense en particulier aux deux sections concernant l'ouverture du naos [42] - ou encore par l'emploi fréquent de la formulation $k y$ r(j)(ky ro) ou «autre formule » qui propose au prêtre de choisir une autre version du même chapitre [43]. De plus, l'étude de la composition a montré que de nombreux passages étaient des emprunts et réutilisation de textes antérieurs. C'est le cas par exemple du chapitre 54 de «présenter l'onguent medjet » qui intègre et reproduit à l'identique les chapitres 72 à 76 des textes des Pyramides. Plus qu'un manuel de conduite du rituel, le papyrus Berlin 3055 est un recueil de formules, laissant libre choix à l'officiant de prononcer l'un ou l'autre chapitre.

On ne peut également manquer de mentionner une composition tout à fait exceptionnelle connue sous l'appellation de Manuel du Temple [44]. Il s'agit d'un texte reproduit sur une quarantaine de manuscrits hiératiques et démotiques et également connu par une version grecque. Il précise l'organisation architecturale du temple et les règles et devoirs des prêtres officiants. Les informations communiquées, en particulier dans la première partie du texte consacrée à la description architecturale, sont souvent à rapprocher d'un temple idéal - à l'image de ce qui est communiqué sur les dimensions et l'organisation interne des temples (nom et disposition des salles), et qui reflète rarement la réalité architecturale de ces édifices. Dans d'autres cas, on constate que le texte faitécho à d'autres sources. Ainsi, des informations relatives au rituel de fondation proposées dans la première partie de la composition peuvent être rapprochées des versions imagées de ce même rituel dans les temples.
[35] ZiegLeR 1984 et ReYNDERS 1998.

[36] Pour Tebtynis, voir Guermeur 2008.

[37] Sur cette question, voir NORDH 1996.

[38] WeSSETZKY 1975 et pour une bibliographie plus à jour, THIERS 2004 , p. 553 , note 6 .

[39] Chassinat 1928a, 3518-9 et Grimm 1989, 160
[40] THIERS 2004, p. 556-560.

[41] Königliche Museen Berlin 1902 et Moret 1902.

[42] MORET 1902, p. 30 et 104.

[43] Ibid., p. 191-197.

[44] Sur le Manuel du Temple, voir Quack 2003, Quack 2004 et Quack 2013. 


\section{LES SOURCES ICONOGRAPHIQUES : LA DÉCORATION DES TEMPLES}

L'Égypte présente la particularité de posséder des temples décorés de scènes rituelles. Ces dernières constituent une source d'information importante sur les rituels. Néanmoins, ce n'est pas la représentation d'un rite sur les murs d'un temple qui en assure la conduite dans l'édifice. En d'autres termes l'image ne fait pas le rite, en revanche l'existence du rite justifie l'image. C'est pour cette raison que nombre de temples dépourvus de décoration ont pu parfaitement remplir leur fonction, le seul élément réellement indispensable étant la statue de culte [45].

Toutefois, l'idéal égyptien veut que le temple soit décoré et que les actions rituelles soient inscrites dans la pierre afin de garantir leur pérennité. La décoration des édifices obéit à un ensemble de principes et de règles conditionnant la mise en image, l'ordonnancement et le placement des scènes [46].

\section{L'image et le rituel}

Les murs des temples égyptiens proposent les versions imagées de nombreux rituels, chaque tableau d'offrande relatant une action [47]. Certains ensembles sont également connus par les versions «papier» ou plutôt «papyrus » des rituels ce qui permet une comparaison des sources. En revanche, si certains rituels ne sont connus que par les versions sur papyrus, de même certaines compositions ne sont attestées que par des tableaux sculptés.

Le rituel divin journalier, qui a été évoqué à plusieurs reprises, comporte, sur papyrus, 65 chapitres. C'est probablement le rituel le plus figuré dans les temples, mais on s'aperçoit que les manières de le retranscrire varient considérablement d'un édifice à l'autre. Ainsi, dans le temple $d^{\prime} E d f o u$, le rituel est le thème de quasiment toutes les scènes décorées du sanctuaire, soit une trentaine de tableaux. À Kom Ombo, il brille par sa discrétion; dans le temple de Philae, il est quasiment inexistant dans le sanctuaire, mais figure dans l'ouâbet. Le nombre de chapitres représentés d'un édifice à l'autre varie, tout comme leur succession sur la paroi et leur positionnement, à l'image de ce qui est observé pour les chapitres 7 à 12 dans quelques grands temples (Fig. 2) [48]. De plus, les textes inscrits sur les parois ne correspondent pas à ceux présents sur le papyrus. Cela s'explique aisément : scène sculptée et papyrus ne jouent pas le même rôle. Le texte sur papyrus est destiné à l'officiant et comporte les formules à réciter. Dans ce contexte, la
Figure 2 : tableau détaillant la représentation et succession de scènes du rituel divin journalier dans quelques grands temples (L. Martzolff).

\begin{tabular}{|l|l|l|l|l|l|}
\cline { 2 - 5 } \multicolumn{1}{l|}{} & Abydos & Louqsor & Philae & Edfou & Dendara \\
\hline Retirer le lien & & & & & \\
\hline $\begin{array}{l}\text { Rompre } \\
\text { I'argile }\end{array}$ & & & & \\
\hline $\begin{array}{l}\text { Délier le } \\
\text { sceau }\end{array}$ & & & & \\
\hline $\begin{array}{l}\text { Découvrir la } \\
\text { face du dieu }\end{array}$ & & & & \\
\hline Voir le dieu & & & & \\
\hline $\begin{array}{l}\text { Renifler la } \\
\text { terre }\end{array}$ & & & & \\
\hline
\end{tabular}

divinité n'a aucun rôle à tenir si ce n'est celui de bénéficiaire. Dans les scènes des temples, la situation est toute différente puisque le tableau sert à évoquer un rituel, témoigne de sa conduite dans le temple, mais ne sert pas de support au rituel. Il met en scène non seulement I'officiant, mais aussi la divinité qui va réagir à l'acte rituel en prononçant des paroles. Ainsi, les thèmes abordés dans l'acte inscrit sur papyrus et leur mise en image sont les mêmes, mais le traitement est différent. L'artiste qui grave la scène sur la paroi doit se plier à une contrainte qui est celle de l'organisation prédéfinie de la scène avec un officiant et une divinité bénéficiaire, ce qui entraîne obligatoirement une adaptation du texte à ce nouveau support. Alors que les inscriptions sur papyrus relèvent du seul ritualiste, dans le temple, l'ensemble du texte de la scène se partageant entre roi et divinité.

Enfin, on observe dans chaque temple une recomposition totale ou partielle du texte de la scène [49]. La comparaison des versions existantes d'un même tableau démontre l'existence de modèles qui se transmettaient au sein des équipes de décorateurs et dont les Maisons de Vie des temples pouvaient posséder des copies. Tous ces éléments expliquent pourquoi, finalement, dans les versions les plus tardives du rituel, le volume de texte reproduit par rapport au papyrus est ridiculement faible. Ainsi à la version « unique » du texte sur papyrus, qui a certes été copiée pour être diffusée, s'opposent les multiples versions des temples.

À l'inverse du rituel divin journalier, qui est connu à la fois par un papyrus et par un nombre très important

[45] TrauneCKer 2005, p. 2135.

[46] Derchain 1962.

[47] Chaque tableau montre le roi ou un officiant en train de faire une action, mais il n'est pas rare que le texte en détaille plusieurs.

[48] MARTZOLFF, à paraître ; Hussy 2007.

[49] Les scènes du temple de Sethi I er en Abydos font figure d'exception car elles sont les seules à présenter sur la paroi la version exacte du texte sur papyrus. Voir, par exemple, les tableaux publiés dans Calverley, Broome \& Gardiner 1933. 
de scènes décorées, les sources relatives au rituel de couronnement du faucon divin se limitent aux textes et images inscrits sur la face interne du mur d'enceinte du grand temple d'Edfou [50]. Si des compositions intégrées à ces scènes - à l'exemple de I'hymne au faucon - sont connues sur certains papyrus comme le papyrus de la confirmation du pouvoir royal [51], on ne possède pas un texte retraçant l'intégralité du rite de couronnement du faucon [52].

Le roi est le représentant d'Horus sur terre. Le rituel de couronnement consacre le renouvellement de la royauté par le truchement de I'animal sacré. Le site d'Edfou est dédié au dieu faucon Horus et comme en d'autres lieux, on y adorait le faucon sacré. Des faucons étaient élevés dans une volière et chaque année, au début du mois de Tybi, la statue du grand temple était emportée en procession dans le temple du faucon sacré, où elle choisissait un animal qui incarnerait le dieu, pour l'année à venir. L'oiseau était bénéficiaire de tout un ensemble de rites et était présenté à la foule avant d'être couronné dans le temple.

Le déroulement du rituel est bien connu et détaillé dans les grands textes qui accompagnent les représentations montrant les sorties en procession du dieu et de son animal sacré sur la face interne du mur d'enceinte du grand temple [53]. La reconstitution du rituel repose presque exclusivement sur les données fournies par la décoration. Il faut ajouter aux scènes de la face nord deux représentations d'intronisation du faucon gravées sur les parois latérales du pylône, représentations qui renvoient sans nul doute au même rituel [54]. Leur présence sur le pylône s'explique d'abord par la nature de l'édifice. Les deux tours ou môles sont identifiées aux deux extrémités de la course solaire et le dieu faucon d'Edfou présente un fort caractère solaire. Ces tableaux encadrent l'espace d'où, en théorie, le soleil émerge à son lever [55]. De plus, cet espace entre les deux môles est qualifié de «fenêtre d'apparition » ou de «fenêtre du faucon-sia » et sert, dans le cadre du rituel de couronnement du faucon, d'espace surélevé pour la présentation de I'animal aux fidèles [56]. Strabon mentionne une pratique identique pour le site méridional de Philae dédié à Isis [57]. C'est d'ailleurs sur le premier pylône de ce temple que se retrouvent des représentations d'intronisation de l'animal identiques à celles observées sur le pylône d'Edfou [58]. En revanche, aucune trace ailleurs dans le temple de représentations du rituel de couronnement. Les scènes du pylône associées au témoignage de Strabon ont servi d'arguments pour justifier l'existence sur le site de Philae d'un rituel du couronnement du faucon. Si I'hypothèse est fort probable, elle doit toutefois être prise en compte avec prudence, d'abord en raison de l'absence de toute autre mention du rituel sur le site, ensuite parce que l'analyse de la décoration des pylônes de Philae fait apparaître de nombreux emprunts décoratifs au pylône d'Edfou [59]. Il faut donc se demander si la gravure de ces scènes, dont la thématique n'est pas incompatible avec la théologie développée à Philae, relève bien d'une volonté d'évoquer et représenter un rituel ou s'il s'agit de reproduire des tableaux décoratifs du pylône d'Edfou qui a pu servir de modèle.

\section{La documentation des cryptes}

À côté des scènes « classiques » recouvrant les murs des salles de culte, se trouve une autre documentation imagée : celle des cryptes décorées. Il s'agit d'une documentation datée principalement de la période tardive et en nombre relativement restreint puisqu'on ne connaît qu'une petite dizaine d'attestations [60]. Les cryptes sont des espaces réservés dont l'accès était caché [61]. Elles servaient principalement à stocker du matériel lorsque ce dernier n'était pas utilisé. Le temple d'Isis à Dendéra est célèbre pour son réseau de cryptes décorées [62]. Beaucoup des scènes d'offrande figurées sont comparables à celles gravées sur les murs des salles du temple. Néanmoins, on y trouve des représentations des objets sacrés du temple ou encore des statues divines. Il est possible de se faire une idée du matériel qui était présent et stocké dans ces espaces non seulement à partir des représentations, mais aussi des textes qui détaillent les dimensions et matériaux utilisés. Une situation comparable
[50] ChASSINAT 1928b, pl. 149 et 154.

[51] Goyon 1972. Une nouvelle édition de ce papyrus est en cours par J.F. Quack (Das Königsritual des Papyrus Brooklyn 47.218.50).

[52] MARTzOLfF 2014, p. 68-69.

[53] Alliot 1954, p. 561-667.

[54] Chassinat 1933 , p. $109^{11}-110^{6}$ et $148^{2-12}$; Bartels 2009 , pl. 19 ; MARTZOLFF 2011, p. 215.

[55] Sur le symbolisme solaire du pylône et I'identification des deux tours aux pôles de la course solaire, voir CHASSINAT 1933, p. 1467-8; MaRTZOLFF 2011, p. 151-152, 199 et 211-213.

[56] Chassinat 1933, p. $110^{2}$ et $148^{12}$.
[57] Strabon, Géographie, XVII, 49.

[58] JUNKER 1958, p. 73-75 et 77-78;MARTZOLFF 2011, p. 87-89.

[59] MARTZOLFF 2011, p. 267-271.

[60] TrauneCKer 1980, col. 825-826; TrauneCKer 1986. Les cryptes sont fréquentes dans les temples égyptiens, mais le nombre de cryptes décorées est faible. On en connaît à Sesebi, dans le temple de Mout à Karnak, à Tôd, Erment, Dendéra, el-Qal'a et dans le temple d'Opet à Karnak.

[61] Voir par exemple la reconstitution d'un système de fermeture pour le temple d'el Qal'a dans Pantalacci \& TrauneCKer 1993, p. 383.

[62] Chassinat 1952 ; Chassinat \& Daumas 1965. 
est observée dans les cryptes d'épaisseur nord et sud du temple d'Opet [63]. Là, les décorateurs y conservaient des statues de mêmes dimensions que les images gravées sur les murs [64].

\section{CONCLUSION}

Étudier le rituel dans le temple égyptienne peut se faire qu'en croisant toutes les informations apportées par les sources archéologiques, papyrologiques ou iconographiques. Si, au premier abord, cet exercice semble facilité par une documentation quasi pléthorique, il rencontre néanmoins de réelles difficultés. En effet, les différentes sources - archéologiques, papyrologiques et iconographiques - qui sont susceptibles de témoigner d'un même rituel ne sont souvent pas contemporaines. Cela n'est pas dû au fait qu'un type de source soit plus particulièrement caractéristique d'une période, mais parce le contexte spécifique à un site (réutilisation des espaces ou à l'inverse abandon brutal) ou encore les aléas des fouilles font que certains secteurs ont fourni principalement une documentation papyrologique - c'est le cas par exemple de Soknepaiou Nesos / Tebtynis [65] alors que d'autres concentrent une documentation iconographique comme les temples d'Edfou ou de Karnak. De plus, chaque documentation doit être analysée en gardant à l'esprit les biais ou limites qui la caractérisent. Pour prendre le seul exemple des tableaux des temples qui représentent des actions rituelles, leur organisation est extrêmement codifiée et soumise à un ensemble de règles qui dictent la mise en image et contraignent le décorateur. Ces contraintes touchent non seulement I'image et son organisation interne (nombre et disposition des protagonistes, gestuelle), mais aussi les textes, leur contenu et leur disposition. Une solide connaissance des spécificités de chaque type de sources mobilisées est donc indispensable dans l'entreprise de reconstitution et d'interprétation des rituels exécutés dans les grands temples égyptiens.

[63] Sur les différents types de cryptes, voir TRAUNECKER 1980.

[64] Traunecker 2004, p. 64.

[65] Voir par exemple Guermeur 2008.

BIBLIOGRAPHIE

Alliot, M., 1949-1954, Le culte d'Horus à Edfou au temps des Ptolémées, 2 vol. (Bibliothèque d'Étude 20), Le Caire.

AMER, H.I., 2012, «Les catacombes osiriennes d'Oxyrynchos », dans L .Coulon (éd.), Le culte d'Osiris au I'er millénaire av. J.-C. (Bibliothèque d'Étude 153), Le Caire, p. 269-282.

Andreu, G., 1997, « Moules d'Osiris », dans G. Andreu, M.-H. Rutschowscaya \& C. Ziegler, L'Égypte ancienne au Louvre, Paris, p. 202-203.

Bartels, U., 2009, Edfu: Die Darstellungen auf den Außenseiten der Umfassungsmauer und auf dem Pylonen. Strichzeichnungen und Photographien (Die Inschriften des Tempels von Edfu, II. Dokumentationen 1), Wiesbaden.

Beinlich, H., 2008, Handbuch der Szenentitel in den Tempeln der griechisch-römischen Zeit Ägyptens, 2 vol. (Studien zu den Ritualszenen altägyptischer Tempel 3), Dettelbach.

Bénédrte, G., 1893, Le temple de Philae (Mémoire publié par les Membres de la Mission Archéologique Française au Caire 13), Le Caire.

Bissing, F. von, 1901, Catalogue général des antiquités du Musée du Caire. Naos, Nr. 3126-3587, Le Caire.

Calverley, A.M., Broome, M.F. \& Gardiner, A.H., 1933, The Tempel of Sethos I at Abydos $I$. The Chapels of Osiris, Isis and Horus, Chicago.

Cauville, S., 1997, Le temple de Dendara X. Les chapelles osiriennes, Le Caire.

Cauville S., 2000, Le temple de Dendara XI, Le Caire.

Cauville S., 2004, Dendara V-VI. Traduction. Les cryptes du temple d'Hathor, 2 vol. (Orientalia Lovaniensia Analecta 131-132), Leuven.

Chassinat, É., 1928A, Le temple d'Edfou III (Mémoire publié par les Membres de la Mission Archéologique Française au Caire 20), Le Caire. 
Chassinat, É., 1928B, Le temple d’Edfou X (Mémoire publié par les Membres de la Mission Archéologique Française au Caire 27), Le Caire.

Chassinat, É., 1933, Le temple d'Edfou VIII (Mémoire publié par les Membres de la Mission Archéologique Française au Caire 25), Le Caire.

Chassinat, É., 1952, Le temple de Dendara V, Le Caire.

Chassinat, É., 1966-1968, Le mystère d'Osiris au mois de Khoiak, 2 vol., Le Caire.

Chassinat, É. \&Daumas, F., 1965, Le temple de Dendara VI, Le Caire.

ContaRd, F., 2009, Il naos di Sethi I da Eliopolis: un monumento per il culto dio Sole (CGT 7002) (Catalogo del Muzeo Egizio di Torino : Serie 1, Monumenti es testi 12), Milano.

Curtis, J. \& Tallis, N (Éd.), 2005, Forgotten Empire: The World of Ancient Persia, London.

Coulon, L., 2011, «Les inscriptions des catacombes osiriennes d'Oxyrhynchos. Témoignages du culte osirien sous les règnes de Ptolémée VI et Ptolémée VIII », dans A. Jördens \& J.F. Quack (éd.), Ägypten zwischen innerem Zwist und äußerem Druck: die Zeit Ptolemaios' VI. bis VIII.Internationales Symposion Heidelberg 16.-19. 9. 2007 (Philippika 45), Wiesbaden, p. 77-91.

Coulon, L., Leclère F. \& MARChand, S., 1995, « «Catacombes» osiriennes de Ptolémée IV à Karnak. Rapport préliminaire de la campagne de fouilles $1993 »$, Cahiers de Karnak 10, p. 205-238.

Davies, N. De Garis, 1953, The Temple of Hibis in El Khargeh Oasis III. The Decoration (Publications of the Metropolitan Museum of Art Egyptian Expedition 17), New York.

Derchain, P., 1962, « Un manuel de géographie liturgique à Edfou », Chronique d'Égypte 37, n73, p. 31-65.

Goyon, J.-C., 1972, La confirmation du pouvoir royal au Nouvel An (Brooklyn Mus. pap. 47.218.50) (Bibliothèque d'Études 52), Le Caire.

Goyon, J.-C., 1975, « Textes mythologiques II. Les révélations du mystère des Quatre Boules », Bulletin de I'Institut français d'Archéologie Orientale 75, p. 349-399.

Goyon, J.-C., 1984, s. v. Räucherung, Lexikon der Ägyptologie V, col. 83-85.

Goyon, J.-C., 1999, Le papyrus d'Imouthès, fils de Psintaês au Metropolitan Museum of Arts de New York (papyrus MMA 35.9.21), New York.

Goyon, J.-C., 2006, Le rituel du sHtp cXmt au changement de cycle annuel (BdÉ 141), Le Caire.

Grimm, A., 1989, « Altägyptische Tempelliteratur. Zur Gliederung und Funktion der Bücherkataloge von Edfu und et-Tôd », dans S. Schoske (Hrsg), Akten des vierten Internationalen Ägyptologen-Kongresses, München 1985 (Beihefte Studien zur altägyptischen Kultur 3), München, p. 159-170.

GuermeUR, I., 2008, « Les nouveaux papyrus hiératiques exhumés sur le site de Tebtynis : un aperçu », dans S. Lippert \& M. Schentuleit (éd.), Graeco-Roman Fayum - Texts and Archaeology. Proceedings of the Third International Fayum Symposion, Freudenstadt, May 29 - June 1, 2007, Wiesbaden, p. 113-122.

HeLck, W., 1980, s. v. Kultstatue, Lexikon der Ägyptologie III, col. 859-863.

HeLcK, W., 1984, s. v. Rituale, Lexikon der Ägyptologie V, col. 119-127.

HöLbL, G., 2004, Altägypten im römischen Reich. Der römische Pharao und seine Tempel. Band II. Die Tempel des römischen Nubien, Mainz am Rhein.

Hussy H., 2007, Die Epiphanie und Erneuerung der Macht Gottes: Szenen des täglichen Kultbildrituals in den ägyptischen Tempeln der griechisch-römischen Epoche (Studien zu den Ritualszenen altägyptischer Tempel 5), Dettelbach.

Junker, H., 1958, Der große Pylon des Tempels der Isis in Philä (Denkschrift der Österreichischen Akademie der Wissenschaften Wien, Sonderband), Wien.

Königliche Museen Berlin, 1902, Rituale für den Kultus des Amon und für den Kultus der Mut (Hieratische Papyrus aus den königlichen Museen zu Berlin), Leipzig.

Leclere, F., 2002, « Fouilles du tombeau d'Osiris à Karnak. Présentation des travaux récents », Bulletin de la Société Française d'Égyptologie 153, p. 24-44.

LoRTon, D., 1999, «The theology of cult statues in Ancient Egypt » dans M.B. Dick (éd.), Born in Heaven, made on Earth: the Making of the Cult Image in the Ancient Near East, Winona Lake, p. 123-210.

MARTzolff, L., 2011, La décoration des pylônes ptolémaïques de Philae et d'Edfou. Étude comparative (Études d'archéologie et d'histoire ancienne), Paris.

MARTzolfF, L., 2014, «Images du rituel de la confirmation du pouvoir royal: le lâcher des oiseaux vivants », dans J.-F. Quack (éd.), Ägyptische Rituale der griechisch-römischen Zeit. Kolloquium Heidelberg, 14-16.07.2008 (Orientalische Religionen in der Antike 6), Tübingen, p.67-81.

MARTzolfF, L., à paraître, « Variations of Tradition. Modalities of ritual adaption in Ancient Egypt II : L'adaptation d'un rituel sur les murs d'un temple à la Période Tardive: I'exemple du rituel divin journalier », dans P. Kousoulis \& N. Lazaridis (éd.), Proceedings of the Tenth International Congress of Egyptologists, University of the Aegean, Rhodes, 22-29 May 2008 (Orientalia Lovaniensia Analacta), Leuven.

Meeks, D., 1976, s. v. Pureté et purification en Égypte, Dictionnaire de la Bible, Supplément IX, col. 430-452.

Moret, A., 1902, Le rituel du culte divin journalier (Annales du Musée Guimet 14), Paris.

Nelson, H.H., 1944, « The Theban Temples of the Empire Period », The Biblical Archaeologist 7/3, p. 44-53.

NordH, K., 1996, Aspects of Ancient Egyptian Curses and Blessings. Conceptual Background and Transmission (Boreas 26), Uppsala.

Oтto, E., 1960, Das ägyptische Mundöffnungsritual (Ägyptologische Abhandlungen 3), Wiesbaden.

Pantalacci, L. \& Traunecker, C., 1993, «Le temple d'el-Qalca à Coptos : état des travaux », Bulletin de I'Institut français d'Archéologie Orientale 93, p. 379-390.

Parker, R.A., Leclant, J., Goyon, J.-C., 1979, The Edifice of Taharqa by the Sacred Lake of Karnak (Brown Egyptological Studies 8), Providence. 
Porter, B. \& Moss, R.L.B., 1972, Topographical Bibliography of Ancient Egyptian Hieroglyphic Texts, Reliefs and Paintings II. Theban Temples, $2^{\mathrm{e}}$ éd. (1 $1^{\text {re }}$ éd. 1929), Oxford.

QuAck, J.F., 2003, «Le Manuel du Temple. Une nouvelle source sur la vie des prêtres égyptiens », Égypte, Afrique \& Orient 29, p. $11-18$.

QuAck, J.F., 2004, «Organiser le culte idéal. Le Manuel du Temple », Bulletin de la Société française d'Égyptologie 160, p. 9-25.

QUACK, J.F., 2005, «Ein Prätext und seine Realisierungen. Facetten des ägyptischen Mundöffnungsrituals », dans B. DüCKER \& H. Roeder (éd.), Text und Ritual. Kulturwissenschaftliche Essays und Analysen von Sesostris bis Dada (Hermeia 8), Heidelberg, p. 165-185.

QuACK, J.F., 2013, « Vom Dekret des Neferkasokar zum Dialog des Imhotep. Ägyptische Textquellen zum idealen Tempel », Sokar 27, p. 64-81.

Quirke, S. \& Spencer, J., 1992, British Museum Book of Ancient Egypt, London.

ReYnders, M., 1998, «sSSt and sxm: names and types of the Egyptian Sistrum », dans W. Clarysse, A. Schoors \& H. Willems (éd.), Egyptian Religion. The Last Thousand Years.Studies Dedicated to the Memory of Jan Quaegebeur (OLA 85), Leuven, p. 1001-1013.

Roeder, G., 1914, Catalogue général des antiquités du Musée du Caire. Naos, Nr. 70001-70050, 2 vol., Le Caire.

Seeber, C., 1980, s. v. Kornosiris, Lexikon der Ägyptologie III, Wiesbaden, col. 744-746.

Sethe, K., 1908, Die altägyptischen Pyramidentexte nach den Papierabdrücken und Photographien des berliner Museums 1, Leipzig.

Thiers, C., 2004, « Fragments de théologies thébaines. La bibliothèque du temple de Tôd », Bulletin de l'Institut français d'Archéologie Orientale104, p. 553-572.

TrauneCKer C., 1972, «Les rites de l'eau à Karnak d'après les textes de la rampe de Taharqa », Bulletin de I'Institut français d'Archéologie Orientale 72, p. 195-236.

Traunecker, C., 1980, s. v. Krypta, Lexikon der ÄgyptologieIII, col. 823-830.

Traunecker, C., 1986, « Cryptes décorées, cryptes anépigraphes », dans Institut d’Égyptologie de Montpellier (éd.), Hommages à François Daumas 2, Montpellier, p. 571-578.

Traunecker, C., 1994, « Cryptes connues et inconnues des temples tardifs », Bulletin de la Société française d'Égyptologie 129, p. 26-36.

Traunecker, C., 2004, « Dimensions réelles et dimensions imaginaires des dieux d'Égypte : les statues secrètes du temple d'Opet à Karnak », Ktèma 29, p. 51-65.

Traunecker C., 2005, s. v. Temple (Égypte), dans J. Leclant (éd.), Dictionnaire de l'Antiquité, Paris, p. 2135-2138.

Wessetzky, V., 1975, s. v. Bibliothek, Lexikon der Ägyptologie I, Wiesbaden, col. 783-785.

WIJngaARden, W. D. van, 1927, «Bestemming en Herkomst van het Monument van Sebekhotep IV », Oudheidkundige mededelingen uit het Rijksmuseum van Oudheden te Leiden 8, p. 14-21.

Wuttmann, M. et alii, 1996, «Premier rapport préliminaire des travaux sur le site de 'Ayn Manawir (oasis de Kharga) », Bulletin de I'Institut français d'Archéologie Orientale 96, p. 385-451.

Wuttmann, M., Coulon, L. \& Gombert, F., 2007, «An assemblage of bronze statuettes in a cult context: the temple of Ayn Manâwir », dans M. Hill \& D. Schorsch (éd.), Gifts for the Gods. Images from Egyptian Temples, New York, p. 167-173.

ZiegleR, C., 1984, s. v.Sistrum, Lexikon der Ägyptologie V, col. 959-963. 